\title{
A 3-Lie algebra and the dIKP Hierarchy
}

Min-Ru Chen, Ying Chen, Zhao-Wen Yan, Jian-Qin Mei, Xiao-Li Wang

To cite this article: Min-Ru Chen, Ying Chen, Zhao-Wen Yan, Jian-Qin Mei, Xiao-Li Wang (2019) A 3-Lie algebra and the dKP Hierarchy, Journal of Nonlinear Mathematical Physics 26:1, 91-97, DOI: https://doi.org/10.1080/14029251.2019.1544791

To link to this article: https://doi.org/10.1080/14029251.2019.1544791

Published online: 04 January 2021 


\title{
A 3-Lie algebra and the dKP Hierarchy
}

\author{
Min-Ru Chen \\ School of Mathematics and Statistics, Henan University, Kaifeng 475004, China \\ Ying Chen \\ School of Mathematical Sciences, Capital Normal University, Beijing 100048, China \\ Zhao-Wen Yan \\ School of Mathematical Sciences, Inner Mongolia University, Hohhot 010021, China \\ Jian-Qin Mei \\ School of Mathematical Sciences, Dalian University of Technology, Dalian 116024, China \\ Xiao-Li Wang \\ School of Mathematics and Statistics, Qilu University of Technology (Shandong Academy of Sciences), Jinan 250353, \\ China \\ wxlspu@qlu.edu.cn
}

Received 18 May 2018

Accepted 15 July 2018

\begin{abstract}
In terms of a 3-Lie algebra and the classical Poisson bracket $\left\{B_{n}, L\right\}$ of the dKP hierarchy, a special 3-bracket $\left\{B_{m}, B_{n}, L\right\}$ is proposed. When $m=0$ or $m=1$, the 3-lax equation $\frac{\partial L}{\partial t}=\left\{B_{m}, B_{n}, L\right\}$ is the dKP hierarchy and the corresponding proof is given. Meanwhile, for the generalized case $(m, n)$, the generalized dKP hierarchy is also investigated.
\end{abstract}

Keywords: 3-Lie algebra; dKP hierarchy; Integrable systems.

2000 Mathematics Subject Classification: 37K10, 35Q53

\section{Introduction}

Nambu mechanics $[14,15]$ is a generalization of classical Hamiltonian mechanics. In the context of integrable systems, the integrable hydrodynamical systems have been investigated via Nambu mechanics $[9,10]$. Moreover various super-integrable systems, such as Calogero-Moser system, Kepler problem, three Hamiltonian structures of Landau problem, have been analyzed in the framework of Nambu mechanics [1,16], where a super-integrable system means that it is not only an integrable system in the Liouville-Arnold sense, but also possesses more constants of motion than degrees of freedom. With the development of infinite-dimensional 3-algebras $[2,3,6,8]$, recently more attempts have been made to understand the connection between the infinite-dimensional 3algebras and the integrable systems in the framework of Nambu mechanics. Chen et al. [4] investigated the classical Heisenberg and $w_{\infty} 3$-algebras, and established the relations between the dispersionless KdV hierarchy and these two infinite-dimensional 3-algebras. They found that the dispersionless KdV system is not only a bi-Hamiltonian system, but also a bi-Nambu-Hamiltonian system. 
The $W_{1+\infty} 3$-algebra was constructed in [5] and its connection with the integrable system has also been investigated.

The dispersionless Kadomtsev-Petviashvili (dKP) hierarchy is a paradigm of the integrable systems, which arises as the quasi classical limit of the KP hierarchy. It consists of an infinite number of nonlinear differential equations. This kind of integrable system was introduced by Lebedev, Manin and Zakharov [13]. Many special solutions were obtained by Kodama and Gibbons [11]. Krichever [12] studied the dKP hierarchy and introduced the analogue of the tau function to integrate the consistency conditions for the free energy of the topological minimal models. It is wellknown that the dKP hierarchy can be represented in terms of a Lax equation $\frac{\partial L}{\partial t_{n}}=\left\{B_{n}, L\right\}$. In this paper, we reinvestigate the property of the Lax equation $B_{n}$ and $L$ of the dKP hierarchy in the framework of 3-Lie algebra. In terms of a 3-bracket and Lax equation of the dKP hierarchy, we present a 3-Lax equation with respect to the Lax triple $\left\{B_{m}, B_{n}, L\right\}$, and derive the corresponding (non)integrable nonlinear evolution equations for the cases of the different Lax triples $\left\{B_{m}, B_{n}, L\right\}$.

This paper is organized as follows. In section 2 , we briefly review the definition of the dKP hierarchy and define a 3-Lax equation $\frac{\partial L}{\partial t_{m n}}=\left\{B_{m}, B_{n}, L\right\}$. Meanwhile, We prove that when $(m, n)$ takes the special values $(0, n+1)$ and $(1, n)$, the dKP hierarchy is derived. In addition, the generalized case of the 3-Lax equation $\frac{\partial L}{\partial t_{m, n}}=\left\{B_{m}, B_{n}, L\right\}$ is discussed. We end this paper with the concluding remarks in section 3 .

\section{The dKP Hierarchy}

The Lax equation with respect to a series of independent time variables $\left(t_{1}, t_{2}, \ldots\right)$ for the dKP hierarchy is

$$
\frac{\partial L}{\partial t_{n}}=\left\{B_{n}, L\right\}, \quad B_{n}=\left(L^{n}\right)_{+},
$$

where $L$ is a Laurent series of $\lambda$ as the form

$$
L=\lambda+\sum_{i=2}^{+\infty} u_{i} \lambda^{-i+1}
$$

$\left(L^{n}\right)_{+}$is the nonnegative powers of $\lambda$ in the Laurent series $L^{n}, u_{i}=u_{i}\left(t_{1}, t_{2}, \ldots\right), i \geqslant 2$, the bracket $\{$,$\} is the Poisson bracket in 2D phase space \left(\lambda, x=t_{1}\right)$

$$
\{f, g\}=\frac{\partial f}{\partial \lambda} \frac{\partial g}{\partial x}-\frac{\partial g}{\partial \lambda} \frac{\partial f}{\partial x} .
$$

The dKP hierarchy (2.1) is a collection of nonlinear differential equations for $u_{n}\left(x, t_{2}, \ldots\right)$ with respect to $\left(x, t_{2}, \ldots\right)$. This system is obtained by replacing microdifferential operators $\partial$ (in $x$ ) and their commutators of the KP hierarchy by Laurent series (in $\lambda$ ) and Poisson brackets. A number of characteristics of the KP hierarchy, indeed, persist in this hierarchy. For example, one can prove that the Lax equations (2.1) are equivalent to the zero-curvature equations

$$
\frac{\partial B_{n}}{\partial t_{m}}-\frac{\partial B_{m}}{\partial t_{n}}+\left\{B_{n}, B_{m}\right\}=0
$$

with purely algebraic manipulation as done for the ordinary KP hierarchy [7]. 
Let us define a 3-bracket

$$
\{f, g, h\}=f\{g, h\}+g\{h, f\}+h\{f, g\},
$$

where $f, g, h$ are smooth functions on $2 \mathrm{D}$ phase space $(\lambda, x)$ and the bracket $\{$,$\} is the Poisson$ bracket (2.3). The 3-bracket (2.4) satisfies the following properties:

1. skew-symmetry: $\left\{f_{\sigma(1)}, f_{\sigma(2)}, f_{\sigma(3)}\right\}=(-1)^{\varepsilon(\sigma)}\left\{f_{1}, f_{2}, f_{3}\right\}$,

2. Fundamental identity:

$$
\left\{f_{1}, f_{2},\left\{f_{3}, f_{4}, f_{5}\right\}\right\}=\left\{\left\{f_{1}, f_{2}, f_{3}\right\}, f_{4}, f_{5}\right\}+\left\{f_{3},\left\{f_{1}, f_{2}, f_{4}\right\}, f_{5}\right\}+\left\{f_{3}, f_{4},\left\{f_{1}, f_{2}, f_{5}\right\}\right\},
$$

where $\varepsilon(\sigma)$ equals to 0 or 1 depending on the parity of the permutation $\sigma$. Therefore, with the 3-bracket (2.4), the set of all the smooth functions on the 2D phase space $(\lambda, x)$ over a field of characteristic zero forms a 3-Lie algebra.

By means of the 3-bracket (2.4), we introduce the 3-Lax equation

$$
\frac{\partial L}{\partial t_{m, n}}=\left\{B_{m}, B_{n}, L\right\}
$$

When $m=0$, it is easy to see that (2.5) becomes (2.1).

Suppose $|\cdot|$ express the weight of $\cdot$. Let us assign the weights as follows,

$$
\left|u_{i}\right|=i(i \geqslant 2), \quad|x|=-1, \quad|1|=0, \quad\left|t_{m, n}\right|=-(m+n),
$$

then we have the following lemma.

Lemma 2.1. For the multivariate polynomial

$$
\begin{aligned}
& (n-1)\left(1+u_{2}+\cdots+u_{n}\right)^{n+1}\left(u_{2}+2 u_{3} \cdots+(n-1) u_{n}\right) \\
& \quad-(n+1)\left(1+u_{2}+\cdots+u_{n}\right)^{n}\left(2 u_{2}+\cdots+n u_{n}\right)+3\left(1+u_{2}+\cdots+u_{n}\right)^{n+1},
\end{aligned}
$$

the terms with weight $n+2(n \geqslant 2)$ disappear.

Proof. The terms with weight $n+2$ in (2.6) can be written as the form $C u_{2}^{i_{2}} u_{3}^{i_{3}} \cdots u_{n}^{i_{n}}$, where $0 \leqslant$ $i_{1}, i_{2}, \ldots, i_{n} \leqslant n$ and $2 i_{2}+3 i_{3}+\cdots+n i_{n}=n+2$. The coefficient $C$ is

$$
\begin{aligned}
C= & (n-1)\left[C_{n+1}^{i_{2}} C_{n+1-i_{2}}^{i_{3}} \cdots C_{n+1-i_{2}-\cdots-i_{n-2}}^{i_{n-1}} C_{n+1-i_{2}-\cdots-i_{n-1}}^{i_{n}-1} \cdot(n-1)\right. \\
& +C_{n+1}^{i_{2}} C_{n+1-i_{2}}^{i_{3}} \cdots C_{n+1-i_{2}-\cdots-i_{n-3}}^{i_{n-2}} C_{n+1-i_{2}-\cdots-i_{n-2}}^{i_{n-1}-1} C_{n+1-i_{2}-\cdots-i_{n-1}+1}^{i_{n}} \cdot(n-2) \\
& \left.+\cdots+C_{n+1}^{i_{2}-1} C_{n+1-i_{2}+1}^{i_{3}} \cdots C_{n+1-i_{2}-\cdots-i_{n-1}+1}^{i_{n}} \cdot 1\right] \\
& -(n+1)\left[C_{n}^{i_{2}} C_{n-i_{2}}^{i_{3}} \cdots C_{n-i_{2}-\cdots-i_{n-2}}^{i_{n-1}} C_{n-i_{2}-\cdots-i_{n-1}}^{i_{n}} \cdot n\right. \\
& +C_{n}^{i_{2}} C_{n-i_{2}}^{i_{3}} \cdots C_{n-i_{2}-\cdots-i_{n-3}}^{i_{n-2}} C_{n-i_{2}-\cdots-i_{n-2}}^{i_{n-1}-1} C_{n-i_{2}-\cdots-i_{n-1}+1}^{i_{n}} \cdot(n-1) \\
& \left.+\cdots+C_{n}^{i_{2}-1} C_{n-i_{2}+1}^{i_{3}} \cdots C_{n-i_{2}-\cdots-i_{n-1}+1}^{i_{n}} \cdot 2\right]+3 C_{n+1}^{i_{2}} C_{n+1-i_{2}}^{i_{3}} \cdots C_{n+1-i_{2}-\cdots-i_{n-1}}^{i_{n}} \\
= & (n-1) \frac{(n+1) !}{i_{2} ! i_{3} ! \cdots i_{n} !\left(n+2-i_{2}-\cdots-i_{n}\right) !} \cdot\left[i_{n}(n-1)+i_{n-1}(n-2)+\cdots+i_{2} \cdot 1\right] \\
& -(n+1) \frac{n !}{i_{2} ! i_{3} ! \cdots i_{n} !\left(n+1-i_{2}-\cdots-i_{n}\right) !}\left[i_{n} n+i_{n-1}(n-1)+\cdots+i_{2} \cdot 2\right] \\
& +3 \frac{(n+1) !}{i_{2} ! i_{3} ! \cdots i_{n} !\left(n+1-i_{2}-\cdots-i_{n}\right) !} .
\end{aligned}
$$


Using the condition $2 i_{2}+3 i_{3}+\cdots+n i_{n}=n+2$, (2.7) equals to

$$
\begin{aligned}
& \frac{(n+1) !}{i_{2} ! i_{3} ! \cdots i_{n} !}\left[\frac{(n-1)\left(n+2-i_{2}-\cdots-i_{n}\right)}{\left(n+2-i_{2}-\cdots-i_{n}\right) !}+\frac{-n-2+3}{\left(n+1-i_{2}-\cdots-i_{n}\right) !}\right] \\
& =\frac{(n+1) !}{i_{2} ! i_{3} ! \cdots i_{n} !}\left[\frac{n-1}{\left(n+1-i_{2}-\cdots-i_{n}\right) !}+\frac{-n+1}{\left(n+1-i_{2}-\cdots-i_{n}\right) !}\right] \\
& =0 .
\end{aligned}
$$

Theorem 2.1. For the 3-brackets $\frac{n-1}{n+1}\left\{B_{0}, B_{n+1}, L\right\}$ and $\left\{B_{1}, B_{n}, L\right\}$, the corresponding coefficients of $\lambda^{-1}$ are the same, i.e. $\frac{n-1}{n+1} \frac{\partial u_{2}}{\partial t_{0, n+1}}=\frac{\partial u_{2}}{\partial t_{1, n}}$ by (2.5). Thus when $m=1$ the 3-Lax equation (2.5) is in fact the $d K P$ hierarchy as the case $m=0$.

Proof. By direct calculations, we obtain

$$
B_{n}=\sum_{\substack{0 \leqslant i_{1}, i_{2}, \ldots, i_{n} \leqslant n \\ i_{1}+i_{2}+\cdots+i_{n}=n \\ 2 i_{2}+3 i_{3}+\cdots+n i_{n} \leqslant n}} C_{n}^{i_{1}} C_{n-i_{1}}^{i_{2}} \cdots C_{n-i_{1}-i_{2}-\cdots-i_{n-1}}^{i_{n}} u_{2}^{i_{2}} u_{3}^{i_{3}} \cdots u_{n}^{i_{n}} \lambda^{n-\left(2 i_{2}+3 i_{3}+\cdots+n i_{n}\right)},
$$

and

$$
\begin{aligned}
& \left\{B_{0}, B_{n+1}, L\right\}=\sum_{\substack{0 \leqslant i_{1}, i_{2}, \ldots, i_{n} \leqslant n \\
i_{1}+i_{2}+\cdots+i_{n}=n \\
2 i_{2}+3 i_{3}+\cdots+n i_{n} \leqslant n}} \sum_{k=2}^{\infty} C_{n+1}^{i_{1}+1} C_{n-i_{1}}^{i_{2}} \cdots C_{n-i_{1}-i_{2}-\cdots-i_{n-1}}^{i_{n}} u_{2}^{i_{2}} u_{3}^{i_{3}} \cdots u_{n}^{i_{n}} u_{k, x} \\
& {\left[(n+1)-\left(2 i_{2}+3 i_{3}+\cdots+n i_{n}\right)\right] \lambda^{n+1-k-\left(2 i_{2}+3 i_{3}+\cdots+n i_{n}\right)}} \\
& -\sum_{\substack{0 \leqslant i_{1}, i_{2}, \ldots, i_{n} \leqslant n \\
i_{1}+i_{2}+\cdots+i_{n}=n \\
2 i_{2}+3 i_{3}+\cdots+n i_{n} \leqslant n+1}} C_{n+1}^{i_{1}+1} C_{n-i_{1}}^{i_{2}} \cdots C_{n-i_{1}-i_{2}-\cdots-i_{n-1}}^{i_{n}} \frac{\partial\left(u_{2}^{i_{2}} u_{3}^{i_{3}} \cdots u_{n}^{i_{n}}\right)}{\partial x} \\
& \lambda^{(n+1)-\left(2 i_{2}+3 i_{3}+\cdots+n i_{n}\right)}-(n+1) u_{n+1, x} \\
& +\sum_{\substack{0 \leqslant i_{1}, i_{2}, \ldots, i_{i} \leqslant n \\
i_{1}+i_{2}+\cdots+i_{n}=n \\
2 i_{2}+3 i_{3}+\cdots+n i_{n} \leqslant n+1}} \sum_{k=2}^{\infty} C_{n+1}^{i_{1}+1} C_{n-i_{1}}^{i_{2}} \cdots C_{n-i_{1}-i_{2}-\cdots-i_{n-1}}^{i_{i_{n}}} \frac{\partial\left(u_{2}^{i_{2}} u_{3}^{i_{3}} \cdots u_{n}^{i_{n}}\right)}{\partial x} u_{k}(k-1) \\
& \lambda^{n+1-k-\left(2 i_{2}+3 i_{3}+\cdots+n i_{n}\right)}+(n+1) u_{n+1, x} \sum_{k=2}^{\infty}(k-1) u_{k} \lambda^{-k} .
\end{aligned}
$$

From the right side of (2.9), the coefficient of $\lambda^{-1}$ in $\frac{n-1}{n+1}\left\{B_{0}, B_{n+1}, L\right\}$ is

$$
(n-1) u_{n+2, x}+\sum_{k=2}^{n} \sum_{\substack{0 \leqslant i_{1}, i_{2}, \ldots, i_{n} \leqslant n \\ i_{1}+i_{2}+\cdots+i_{n}=n \\ 2 i_{2}+3 i_{3}+\cdots+n i_{n}=n+2-k}} \frac{n-1}{i_{1}+1} C_{n}^{i_{1}} C_{n-i_{1}}^{i_{2}} \cdots C_{n-i_{1}-i_{2}-\cdots-i_{n-1}}^{i_{n}}(k-1) \frac{\partial\left(u_{2}^{i_{2}} u_{3}^{i_{3}} \cdots u_{n}^{i_{n}} u_{k}\right)}{\partial x}
$$


Similarly, the coefficient of $\lambda^{-1}$ in $\left\{B_{1}, B_{n}, L\right\}$ is

$$
\begin{gathered}
(n-1) u_{n+2, x}+\sum_{k=2}^{n} \sum_{\begin{array}{c}
0 \leqslant i_{1}, i_{2}, \ldots, i_{n} \leqslant n \\
i_{1}+i_{2}+\cdots+i_{n}=n \\
2 i_{2}+3 i_{3}+\cdots+n i_{n}=n+2-k
\end{array}} C_{n}^{i_{1}} C_{n-i_{1}}^{i_{2}} \cdots C_{n-i_{1}-i_{2}-\cdots-i_{n-1}}^{i_{n}} k \frac{\partial\left(u_{k} u_{2}^{i_{2}} u_{3}^{i_{3}} \cdots u_{n}^{i_{n}}\right)}{\partial x} \\
-3 \sum_{k=2}^{n} \sum_{\substack{0 \leqslant i_{1}, i_{2}, \ldots, i_{n} \leqslant n \\
i_{1}+i_{2}+\cdots+i_{n}=n \\
2 i_{2}+3 i_{3}+\cdots+n i_{n}=n+2-k}} C_{n}^{i_{1}} C_{n-i_{1}}^{i_{2}} \cdots C_{n-i_{1}-i_{2}-\cdots-i_{n-1}}^{i_{n}} u_{k, x} u_{2}^{i_{2}} u_{3}^{i_{3}} \cdots u_{n}^{i_{n}} .
\end{gathered}
$$

To prove the theorem, we need prove the following equality

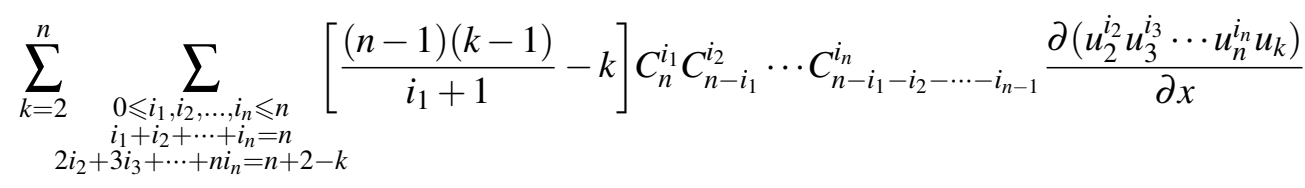

$$
\begin{aligned}
& +3 \sum_{k=2}^{n} \sum_{\substack{0 \leqslant i_{1}, i_{2}, \ldots, i_{n} \leqslant n \\
i_{1}+i_{2}+\cdots+i_{n}=n \\
2 i_{2}+3 i_{3}+\cdots+n i_{n}=n+2-k}} C_{n}^{i_{1}} C_{n-i_{1}}^{i_{2}} \cdots C_{n-i_{1}-i_{2}-\cdots-i_{n-1}}^{i_{n}} u_{k, x} u_{2}^{i_{2}} u_{3}^{i_{3}} \cdots u_{n}^{i_{n}}=0 .
\end{aligned}
$$

On the left side of (2.12), the first term is the terms with weight $n+3$ among

$$
\begin{aligned}
\frac{\partial}{\partial x} & {\left[\sum_{k=2}^{n} \sum_{0 \leqslant i_{1} \leqslant n+1} \frac{n-1}{n+1} C_{n+1}^{i_{1}}\left(u_{2}+\cdots+u_{n}\right)^{n+1-i_{1}}(k-1) u_{k}\right.} \\
& \left.-\left(1+u_{2}+\cdots+u_{n}\right)^{n}\left(2 u_{2}+\cdots+n u_{n}\right)\right] \\
= & \frac{\partial}{\partial x}\left[\frac{n-1}{n+1}\left(1+u_{2}+\cdots+u_{n}\right)^{n+1}\left(u_{2}+2 u_{3} \cdots+(n-1) u_{n}\right)\right. \\
& \left.-\left(1+u_{2}+\cdots+u_{n}\right)^{n}\left(2 u_{2}+\cdots+n u_{n}\right)\right] .
\end{aligned}
$$

Meanwhile the second term is the terms with weight $n+3$ among

$$
3\left(1+u_{2}+\cdots+u_{n}\right)^{n}\left(u_{2, x}+\cdots+u_{n, x}\right)=\frac{3}{n+1} \frac{\partial}{\partial x}\left[\left(1+u_{2}+\cdots+u_{n}\right)^{n+1}\right] .
$$

So the proof of (2.12) is equivalent to the proof of the terms with weight $n+2$ among

$$
\begin{aligned}
& (n-1)\left(1+u_{2}+\cdots+u_{n}\right)^{n+1}\left(u_{2}+2 u_{3} \cdots+(n-1) u_{n}\right) \\
& -(n+1)\left(1+u_{2}+\cdots+u_{n}\right)^{n}\left(2 u_{2}+\cdots+n u_{n}\right)+3\left(1+u_{2}+\cdots+u_{n}\right)^{n+1}
\end{aligned}
$$

disappear, which follows from Lemma 2.1.

We further calculate the more general case of the 3-Lax equation (2.5). For convenience, let $I$ represent the condition $0 \leqslant i_{1}, \ldots, i_{m} \leqslant m ; i_{1}+i_{2}+\cdots+i_{m}=m ; 2 i_{2}+3 i_{3}+\cdots+m i_{m} \leqslant m, J$ represent $0 \leqslant j_{1}, \ldots, j_{n} \leqslant n ; j_{1}+j_{2}+\cdots+j_{n}=n ; 2 j_{2}+3 j_{3}+\cdots+n j_{n} \leqslant n$ and $K$ represent the condition $\left(2 i_{2}+\cdots+m i_{m}\right)+\left(2 j_{2}+\cdots+n j_{n}\right)=m+n-k+1$. 
Substituting (2.8) into (2.5) and comparing the coefficient of $\lambda^{-1}$ on both sides, we obtain

$$
\begin{aligned}
\frac{\partial u_{2}}{\partial t_{m, n}}= & (n-m) u_{m+n+1, x}+\sum_{k=2}^{m+n} \sum_{I, J, K} C_{m}^{i_{1}} C_{m-i_{1}}^{i_{2}} \cdots C_{m-i_{1}-\cdots-i_{m-1}}^{i_{m}} C_{n}^{j_{1}} C_{n-j_{1}}^{j_{2}} \\
& \cdots C_{n-j_{1}-\cdots-j_{n-1}}^{j_{n}}\left\{\left[u_{k}\left(u_{2}^{i_{2}} \cdots u_{m}^{i_{m}}\right)\left(u_{2}^{j_{2}} \cdots u_{n}^{j_{n}}\right)\right]_{x}\left[-n-k+1+\left(2 j_{2}+\cdots+n j_{n}\right)\right]+3(k-1)\right. \\
& \left.u_{k}\left(u_{2}^{i_{2}} \cdots u_{m}^{i_{m}}\right)\left(u_{2}^{j_{2}} \cdots u_{n}^{j_{n}}\right)_{x}+3\left[n-\left(2 j_{2}+\cdots+n j_{n}\right)\right] u_{k, x}\left(u_{2}^{i_{2}} \cdots u_{m}^{i_{m}}\right)\left(u_{2}^{j_{2}} \cdots u_{n}^{j_{n}}\right)\right\} .
\end{aligned}
$$

Similarly, we obtain

$$
\begin{gathered}
\frac{\partial u_{2}}{\partial t_{0, m+n}}=(m+n) u_{m+n+1, x}+\sum_{k=2}^{m+n} \sum_{\begin{array}{c}
0 \leqslant i_{1}, \ldots, i_{m+n} \leqslant m+n \\
i_{1}+i_{2}+\cdots+i_{m+n}=m+n \\
2 i_{2}+\cdots+(m+n) i_{m+n}=m+n+1-k
\end{array}} C_{m+n}^{i_{1}} C_{m+n-i_{1}}^{i_{2}} \cdots \\
C_{m+n-i_{1}-\cdots-i_{m+n-1}}^{i_{m+n}}(k-1)\left(u_{k} u_{2}^{i_{2}} \cdots u_{m+n}^{i_{m+n}}\right)_{x} .
\end{gathered}
$$

When $m=1$, as the result in the Theorem 2.1, (2.15) and (2.16) support $\frac{n-1}{n+1} \frac{\partial u_{2}}{\partial t_{0, n+1}}=\frac{\partial u_{2}}{\partial t_{1, n}}$. But for the general case of $m, n \geqslant 2$ and $m \neq n$, there is not such kind of the equivalent relation between $\frac{\partial u_{2}}{\partial t_{0, m+n}}$ and $\frac{\partial u_{2}}{\partial t_{m, n}}$.

Substituting (2.8) into (2.5), when $m=0, n=2$, we obtain

$$
\sum_{i=1}^{\infty} \frac{\partial u_{i+1}}{\partial t_{02}} \lambda^{-i}=2 u_{3, x} \lambda^{-1}+\sum_{i=2}^{\infty} 2\left[u_{i+2, x}+(i-1) u_{i} u_{2, x}\right] \lambda^{-i}
$$

Comparing the coefficient of $\lambda^{-i}, i \geqslant 1$ on both sides of (2.17), we obtain

$$
\frac{\partial u_{2}}{\partial t_{0,2}}=2 u_{3, x}, \quad \frac{\partial u_{i+1}}{\partial t_{0,2}}=2 u_{i+2, x}+2(i-1) u_{i} u_{2, x}, \quad i \geqslant 2 .
$$

Let $t_{02}=y,(2.18)$ gives the recursion relations

$$
\begin{aligned}
u_{3} & =\frac{1}{2} \partial_{x}^{-1} u_{2, y}, \\
u_{i+2} & =\frac{1}{2} \partial_{x}^{-1} u_{i+1, y}-(i-1) \partial^{-1}\left(u_{i} u_{2, x}\right), \quad i \geqslant 2 .
\end{aligned}
$$

Substituting (2.19) for the case $m=0, n \geqslant 3$ and $m=1, n \geqslant 2$ into (2.15), we get the dKP hierarchy. Substituting (2.19) for the case $m, n \geqslant 1$ and $m \neq n$ into (2.15), we get a general hierarchy. The properties of the general hierarchy deserve our further study.

\section{Summary}

By combining the Lax equation of the dKP hierarchy and the 3-bracket, we have defined a Lax triple $\left\{B_{m}, B_{n}, L\right\}$ and investigated the relations between different pairs of $(m, n)$. Here the 3-bracket is not a Nambu-Poisson bracket, but it satisfies the conditions of 3-Lie algebra. We have proved that when $m=0$ and $m=1$, the 3 -Lax equation $\frac{\partial L}{\partial t_{m, n}}=\left\{B_{m}, B_{n}, L\right\}$ is the dKP hierarchy. For the generalized case of $(m, n)$, the 3-Lax equation means a generalized dKP hierarchy including the dKP hierarchy. Whether the hierarchy of the general case constitute a new integrable hierarchy remains to be studied. 
Our analyses suggest that there exists much deeper connections between the infinitedimensional 3-algebra and the integrable system. More properties with respect to their relations still deserve further study. We believe that 3-algebras may shed new light on the integrable systems.

\section{Acknowledgments}

The authors are grateful to Professor Wei-Zhong Zhao for his valuable discussions and suggestions. We also thank Doctor Chun-Hong Zhang and Rui Wang for their discussions during our calculations. This work is partially supported by National Science Foundation (NSF) projects (Grant Nos. 11505046, 11605096 and 11801292).

\section{References}

[1] J.F. Carinena, P. Guha and M.F. Ranada, Hamiltonian and quasi-Hamiltonian systems, Nambu-Poisson structures and symmetries, J. Phys. A: Math. Theor. 41 (2008) 335209.

[2] S. Chakrabortty, A. Kumar and S. Jain, $w_{\infty}$ 3-algebra, JHEP 09 (2008) 091.

[3] M.R. Chen, K. Wu and W.Z. Zhao, Super $w_{\infty}$ 3-algebra, JHEP 09 (2011) 090.

[4] M.R. Chen, S.K. Wang, K. Wu and W.Z. Zhao, Infinite-dimensional 3-algebra and integrable system, JHEP 12 (2012) 030.

[5] M.R. Chen, S.K. Wang, X.L. Wang, K. Wu and W.Z. Zhao, On $W_{1+\infty} 3$-algebra and integrable system, Nucl. Phys. B 891 (2015) 655-675.

[6] T.L. Curtright, D.B. Fairlie and C.K. Zachos, Ternary Virasoro-Witt algebra, Phys. Lett. B 666 (2008) 386.

[7] E. Date, M. Jimbo, M. Kashiwara and T. Miwa, in Nonlinear Integrable Systems, M. Jimbo and T. Miwa (eds.) (World Scientific, Singapore, 1983).

[8] L. Ding, X.Y. Jia, K. Wu, Z.W. Yan and W.Z. Zhao, On q-deformed infinite-dimensional n-algebra, Nucl. Phys. B 904 (2016) 18-38.

[9] P. Guha, Volume preserving multidimensional integrable systems and Nambu-Poisson geometry, $J$. Nonlinear Math. Phys. 8 (2001) 325-341.

[10] P. Guha, Applications of Nambu mechanics to systems of hydrodynamical type II, J. Nonlinear Math. Phys. 11 (2004) 223-232.

[11] Y. Kodama, A method for solving the dispersionless KP equation and its exact solutions, Phys. Lett. A 129 (1988), 223-226; Y. Kodama and J. Gibbons, A method for solving the dispersionless KP hierarchy and its exact solutions, II, Phys. Lett. A 135 (1989), 167-170; Y. Kodama, Solutions of the dispersionless Toda equation, Phys. Lett. A 147 (1990), 477-482.

[12] I.M. Krichever, The dispersionless Lax equations and topological minimal models, Commun. Math. Phys. 143 (1991), 415-426; The $\tau$-Function of the Universal Whitham Hierarchy, Matrix Models and Topological Field Theories, LPTENS- 92-18, hep-th/9205110, to appear in: Commun. Pure and Appl. Math., XLVII (1994).

[13] D. Lebedev and Yu. Manin, Conservation Laws and Lax Representation on Bennys Long Wave Equations, Phys. Lett. A 74 (1979), 154-156; V.E. Zakharov, On the Benneys equations, Physica D 3 (1981), 193-202.

[14] Y. Nambu, Generalized Hamiltonian Dynamics, Phys. Rev. D 7 (1973) 2405-2412.

[15] L. Takhtajan, On foundation of the generalized Nambu mechanics, Commun. Math. Phys. 160 (1994) 295-315.

[16] A. Tegmen and A. Vercin, Superintegrable systems, multi-hamiltonian structures and nambu mechanics in an arbitrary dimension, Int. J. Mod. Phys. A 19 (2004) 393-409. 